Delft University of Technology

\title{
Decoupling Edge Versus Bulk Conductance in the Trivial Regime of an InAs/GaSb Double Quantum Well Using Corbino Ring Geometry
}

Nguyen, Binh Minh; Kiselev, Andrey A.; Noah, Ramsey; Yi, Wei; Qu, Fanming; Beukman, Arjan J.A.; De Vries, Folkert K.; Van Veen, Jasper; Nadj-Perge, Stevan; Kouwenhoven, Leo P.

DOI

10.1103/PhysRevLett.117.077701

Publication date

2016

Document Version

Final published version

Published in

Physical Review Letters

\section{Citation (APA)}

Nguyen, B. M., Kiselev, A. A., Noah, R., Yi, W., Qu, F., Beukman, A. J. A., De Vries, F. K., Van Veen, J., Nadj-Perge, S., Kouwenhoven, L. P., Kjaergaard, M., Suominen, H. J., Nichele, F., Marcus, C. M., Manfra, M. J., \& Sokolich, M. (2016). Decoupling Edge Versus Bulk Conductance in the Trivial Regime of an InAs/GaSb Double Quantum Well Using Corbino Ring Geometry. Physical Review Letters, 117(7), [077701]. https://doi.org/10.1103/PhysRevLett.117.077701

Important note

To cite this publication, please use the final published version (if applicable).

Please check the document version above. 


\title{
Decoupling Edge Versus Bulk Conductance in the Trivial Regime of an InAs/GaSb Double Quantum Well Using Corbino Ring Geometry
}

\author{
Binh-Minh Nguyen, ${ }^{1, *}$ Andrey A. Kiselev, ${ }^{1}$ Ramsey Noah, ${ }^{1}$ Wei Yi, ${ }^{1}$ Fanming Qu, ${ }^{2}$ Arjan J. A. Beukman, ${ }^{2}$ \\ Folkert K. de Vries, ${ }^{2}$ Jasper van Veen, ${ }^{2}$ Stevan Nadj-Perge, ${ }^{2}$ Leo P. Kouwenhoven, ${ }^{2}$ Morten Kjaergaard, ${ }^{3}$ \\ Henri J. Suominen, ${ }^{3}$ Fabrizio Nichele, ${ }^{3}$ Charles M. Marcus, ${ }^{3}$ Michael J. Manfra, ${ }^{4}$ and Marko Sokolich ${ }^{1, \dagger}$ \\ ${ }^{1}$ HRL Laboratories, 3011 Malibu Canyon Road, Malibu, California 90265, USA \\ ${ }^{2}$ QuTech and Kavli Institute of Nanoscience, Delft University of Technology, 2600 GA Delft, The Netherlands \\ ${ }^{3}$ Center for Quantum Devices, Niels Bohr Institute, University of Copenhagen, 2100 Copenhagen, Denmark \\ ${ }^{4}$ Department of Physics and Astronomy, and Station Q Purdue, Purdue University, West Lafayette, Indiana 47907, USA
}

(Received 2 May 2016; published 12 August 2016)

\begin{abstract}
A Corbino ring geometry is utilized to analyze edge and bulk conductance of InAs/GaSb quantum well structures. We show that edge conductance exists in the trivial regime of this theoretically predicted topological system with a temperature-insensitive linear resistivity per unit length in the range of $2 \mathrm{k} \Omega / \mu \mathrm{m}$. A resistor network model of the device is developed to decouple the edge conductance from the bulk conductance, providing a quantitative technique to further investigate the nature of this trivial edge conductance, conclusively identified here as being of $n$ type.
\end{abstract}

DOI: 10.1103/PhysRevLett.117.077701

InAs/GaSb double-quantum-well structures have been long known to exhibit semimetallic behavior when the electron energy level in the InAs well lies below the hole energy level in the GaSb well [1-6]. In such an "inverted" regime, the material system was predicted to be a topological quantum spin Hall insulator with insulating bulk and conducting helical edge states [7]. This theoretical proposal opened a new prospect for the double-quantum-well structure to be used in Majorana fermion devices and quantum computing [8]. Yet, it remains a great challenge to reliably identify the inverted regime and reveal its helical edge states.

As the electronic structure of the InAs/GaSb quantum wells can be continuously tuned under the electrostatic action of top and bottom gates, the most straightforward way to identify the inverted regime is to construct a $2 \mathrm{D}$ phase diagram as a function of top and back gate biases similar to what has been theoretically predicted [7]. Shown as an example in Fig. 1(a) is the phase diagram of the InAs/GaSb device as a function of back gate bias $\left(V_{\mathrm{BG}}\right)$ and top gate bias $\left(V_{\mathrm{TG}}\right)$, calculated using the capacitor model that we have introduced in Ref. [9] (see, specifically, its Supplemental Material, Sec. III for details). Ideally, one would expect to be able to navigate in the gate bias space between the topological regime where electrons and holes coexist [region I in Fig. 1(a)] and the trivial regime where both type of carriers are depleted [region II in Fig. 1(a)]. However, in many cases, the weak and leaky backgate action due to a thick, defective buffer layer limits the range of operation of the back gate, thus hindering the ability to construct this $2 \mathrm{D}$ phase diagram. Without a complete $2 \mathrm{D}$ phase diagram, the inverted regime can only be indirectly inferred, e.g., based on the resistance behavior under in-plane $[3,10]$ or out of plane $[10,11]$ magnetic field. It was not until recently that the complete 2D phase diagram was experimentally demonstrated [9] with the use of high mobility materials and efficient back gate coupling [12].

To date, multiple approaches have been utilized to capture the signature of edge conductance in InAs/ $\mathrm{GaSb}$ quantum well devices. The most common technique is based on nonlocal measurement of a Hall bar device which shows a nonlocal resistance, sometimes close to the theoretically predicted quantized resistance values [13-18]. Edge mode transport was also deduced from superconducting quantum interference patterns in a superconductor-InAs/GaSb-supercondu+ctor Josephson junction device [19]. Magnetic flux image reconstruction employing a superconductor quantum interference device (SQUID) can also visualize edge conductance in an InAs/GaSb Hall bar [20]. Nevertheless, Refs. [13-20] are lacking in providing clear evidence that these reported edge transports were observed in the inverted regime. In lieu, given the existence of the edge conductance, it is taken for granted that the device is in the inverted regime. However, the observed resistance peak (RP) was found to be insensitive to the in-plane magnetic field [16,19], which contradicts the inverted regime identification criterion $[3,10]$ and requires further theoretical hypotheses [21]. Very recently, Nichele et al. [22] reported that edge conductance is present in the trivial regime, as observed in both transport and scanning SQUID microscopy. In this report, we further substantiate this claim with analysis of a novel Corbino ring device, confirming the presence of edge channels in the trivial regime. In addition, the edge and bulk resistivities have been extracted and mapped in a two dimensional gate bias space of the top and back gates, 
revealing the $n$-type nature of the edge conductance in the trivial regime.

In order to decouple the edge and bulk contributions, we utilized a Corbino-type device geometry allowing for a measurement setup without conductance paths attainable via an edge channel alone. In addition, we chose a Corbino ring geometry with multiple inner and outer pairs of contact leads [Fig. 1(b)] in order to isolate the contribution of contact resistance that is unavoidable in a solid disk Corbino device with only two terminals [16]. This allows for reliable measurements further outside of the RPs where the device resistance is extremely small and cannot be measured in a Corbino disk geometry. Indeed, in this multiple-lead configuration of Corbino ring devices, nonlocal measurement is possible, similar to multilead Hall bars, but with the added advantage of allowing both "with-edge" configurations, i.e., those containing conductance paths attainable via an edge channel alone, and "no-edge" ones (with no such paths) on the same device. This is critical for quantitative extraction of edge and bulk resistivity, especially at the RPs where the bulk resistance is very high compared to edge resistance. Transport at the RPs in Hall bar-type devices is dominated by edge transport $[19,20]$, making it hard to deduce the bulk
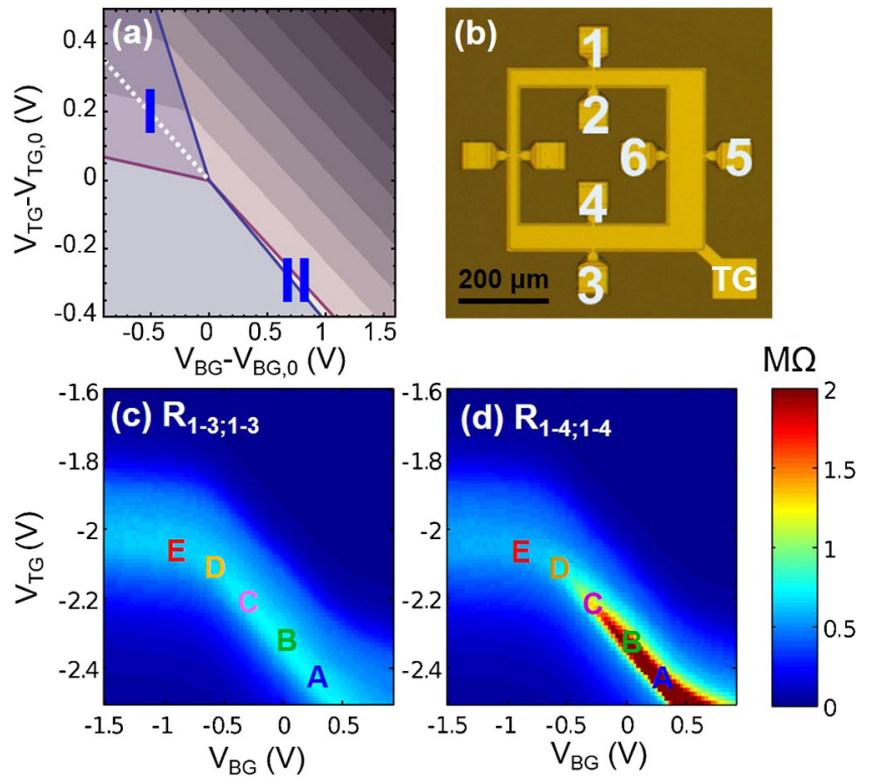

FIG. 1. (a) Simulated phase diagram (based on actual device geometries-InAs-on-GaSb heterostack reported in Ref. [12] with a $40 \mathrm{~nm} \mathrm{HfO}_{2}$ top gate dielectric) illustrating the position of two regimes: inverted charge neutrality point (region I-dashed line) and trivial resistance peak (region II with narrow white sector). The contour lines in the electron-rich sectors mark constant electron densities at multiples of $9.66 \times 10^{10} \mathrm{~cm}^{-2}$, corresponding, at $B=2 \mathrm{~T}$, to filling of complete Landau levels (b) optical micrograph of a Corbino ring device with top gate lead. Resistance maps at $1.8 \mathrm{~K}$ for (c) with edge $R_{1-3 ; 1-3}$ and (d) no edge $R_{1-4 ; 1-4}$ (see text for a definition). Points $\mathrm{A}-\mathrm{E}$ in (c),(d) indicate gate biases where temperature dependent measurements were performed (see Supplemental Material [24], Sec. II). contribution [13-17]. Whereas, for Corbino ring devices, the interplay between bulk and edge conductance can be clearly observed and extracted from the same device, which can eliminate processing uncertainty causing device-todevice variation. As will be detailed in this report, analysis of a Corbino ring device provides a simple yet effective method to extract the contribution of edge conductance, similar to what could be achieved by more elaborated SQUID measurements.

Devices used in this work were grown and fabricated using the same procedure as the previous study [12]. The only difference is that the wafer was grown in a different growth campaign, and resulted in slightly lower mobility, $200000 \mathrm{~cm}^{2} / \mathrm{Vs}$ in this material, in comparison with $500000 \mathrm{~cm}^{2} / \mathrm{Vs}$ in our previously reported results [12], both at $N_{s}=10^{12} \mathrm{~cm}^{-2}$. It is important to note that this lower mobility is comparable to values reported by others $[4,10,11]$ and that lowering mobility, by intentionally introducing disorder, $[16,23]$ is generally accepted as a means to suppress bulk conductivity. Figure 1(b) shows an optical micrograph of the Corbino ring device with inner and outer square size of 340 and $440 \mu \mathrm{m}$, respectively. Channel widths between pairs 1-2, 3-4, and 5-6 are 40, 60, and $80 \mu \mathrm{m}$, respectively. The left pair of leads with $20 \mu \mathrm{m}$ separation was not used in this study. All data were collected at $1.8 \mathrm{~K}$ (except for the temperature dependent measurements presented in the Supplemental Material [24]).

Figures 1(c) and 1(d) show the 2D resistance maps for the with-edge $R_{1-3 ; 1-3}$ and no-edge $R_{1-4 ; 1-4}$, respectively. The notation $R_{i-j ; k-l}$ indicates current fed through leads $i$ and $j$ and voltage recorded between leads $k$ and $l$. Despite having nominally the same structural design, lower mobility wafers in this growth campaign consistently show only one branch of the phase diagram while the high mobility materials [12] exhibited a clear delineation between the inverted and the normal regimes (see Supplemental Material [24], Fig. S1 and Ref. [9]). Devices of limited tunability were also reported in Refs. [15,19] where edge conductance was shown to exist. The correlation between mobility and observation of the inverted regime in the 2D phase diagram is still under investigation, but is beyond the scope of this report, which highlights the presence of edge conductance in the trivial regime.

Comparing Figs. 1(c) and 1(d), a clear distinction between with-edge and no-edge peak resistances (e.g., $7 \times 10^{5} \Omega$ for $R_{1-3 ; 1-3}$ vs $4 \times 10^{6} \Omega$ for $R_{1-4 ; 1-4}$ at the same point A) is a strong evidence for edge conductance. This is not entirely unexpected as it is well known that the free surface of InAs creates an accumulation channel for electrons [27-31]. Since the no-edge resistance is a few times larger than the with-edge one, we conclude that $\mathrm{R}_{1-3 ; 1-3}$ is mostly due to the outer mesa edges of $880 \mu \mathrm{m}$ length (with 2 edges in parallel), giving a rough estimate of $1.6 \mathrm{k} \Omega / \mu \mathrm{m}$ for the linear edge resistivity per micron (Fig. S4). This number matches with the length normalization from inner path 
$R_{2-4 ; 2-4}$ and a Hall bar geometry (Fig. S5). This is also in agreement with what we get from a more quantitative extraction, as will be shown later.

With the InAs-on-GaSb heterostack [12] used in this work, the back gate acts primarily as the hole gate, and the top gate acts as the electron gate. The bottom right corner of the phase diagram corresponds to the depletion of both electron (negative top gate) and hole (positive back gate), suggesting the RPs we are observing are those of the trivial regime for the measured gate bias range. The arrow shape of the RP pointing toward the top left corner also indicates that the gap is closing, similar to what one would expect theoretically [Fig. 1(a) and Ref. [7]]. The gap closure is also confirmed by the temperature dependence of RP with and without edge, as shown in Fig. S2.

Evidence for edge conductance is reinforced with the local and non-local resistance measurement, as shown in Fig. 2 and Supplemental Material [24], Sec. V. Despite the fact that the current is fed through one pair of opposite leads (through 1-2 leads in Fig. 2, or through 3-4 or 5-6 in Fig. S6), at RPs, a substantial voltage drop is measured across the other pairs. This can only be explained if the edge conductance is significant, forming a quasiequipotential line along the device edge. While the resistance at peaks are similar, outside of the RP, the resistance in the electron regime (top right side of the RP ridge) is in the range of $10^{3} \Omega$ for diagonal resistance $R_{1-2 ; 1-2}$, $R_{3-4 ; 3-4}, R_{5-6 ; 5-6}$, but is in the noise floor (a few ohms) for $R_{1-2 ; 3-4}$ and $R_{1-2 ; 5-6}$. This is due to the presence of non-negligible contact resistance (sub-k $\Omega$ range) when the channel resistance (few $\Omega$ ) is low. Even if contact resistance were completely eliminated, the low bulk resistance would make the current flow most directly between leads 1 and 2, resulting in a progressively lower voltage buildup between the other (left floating) leads, (see Supplemental Material [24], Sec. III). This prevents quantitative analysis of edge vs bulk contribution further outside the RP. Nevertheless, using nonlocal measurements we are able to extend accessible dynamic range at least by an order of magnitude.

To highlight the superior dynamic range of this novel Corbino ring design over conventional Hall bars and Cobino disk, we apply a perpendicular magnetic field and document a formation of the integer quantum Hall effect (IQHE). Bulk resistivity is enhanced due to formation of the localized Landau orbitals. At $B=2 \mathrm{~T}$ [Figs. 2(e)-2(h), and $B=1 \mathrm{~T}$ in supporting Fig. S8], the resistance map of $R_{1-2 ; 1-2}$ does not seem to change much because it is still dominated by contact resistance outside of the RP, oscillations only appear in the top right corner with high electron density. However, in nonlocal measurement $R_{1-2 ; 3-4}$ and $R_{1-2 ; 5-6}$, where contact resistance is eliminated, with the resistance lifted from the noise floor only to $\sim 10^{2} \Omega$ range, the oscillations are clearly visible and appear much closer to the RP. It is worth noting that the oscillations in two separate measurements of $R_{1-2 ; 3-4}$ and $R_{1-2 ; 5-6}$ are perfectly in phase, indicating great measurement stability with no shift or drift with gate bias within one cooldown cycle. More interestingly, between the RP and the electron-rich resistance plateau, there is a "canyon" of lower resistance, which cannot be observed in direct measurement of $R_{1-2 ; 1-2}$ [Figs. 2(d),2(h)]. This resistance canyon and plateau come from a crossover between disorder-limited bulk conduction and gradual formation of the IQHE regime as the device disorder is progressively screened by increasing carrier density. Indeed, close to the RP, behavior at finite magnetic field is similar to the $B=0$ case as material is too disordered for IQHE. Even though finite $B$ is kept unchanged, as carrier density increases, screening of disorder improves, leading to better localization of carriers by magnetic field, suppression of bulk conduction and formation of (semi-ballistic) chiral edge states. We stress that these IQHE protected edge states introduce a parallel edge conduction of a completely
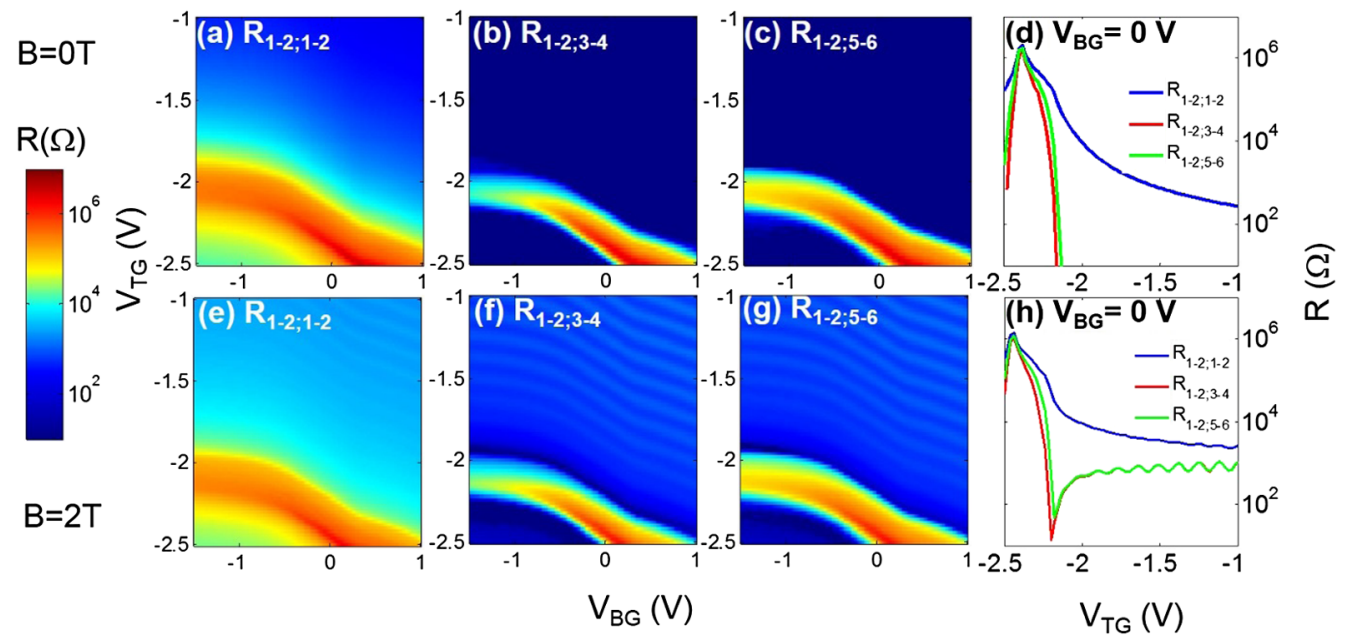

FIG. 2. Local and nonlocal resistance maps (measured at $1.8 \mathrm{~K}$ ) at $B=0 \mathrm{~T}$ (a),(b),(c) and $2 \mathrm{~T}$ (e),(f),(g) indicating an edge conductance path at the RPs; (d) and (h) are $1 \mathrm{D}$ line cuts at $V_{\mathrm{BG}}=0 \mathrm{~V}$. 
different nature (although both are likely facilitated by potential bending near edges). Ripplings in resistance maps manifest consecutive filling of disorder-broadened Landau levels (LL) in the bulk of the device, also accompanied by creation, one by one, of additional chiral edge channels. With electron density of $9.66 \times 10^{10} \mathrm{~cm}^{-2}$ per single LL (when accounting for two spin subbands), and filling of up to about 10 individual LLs visible, Figs. 2(f) and 2(g) present a very detailed 2D gate bias map of the electron density. Selfconsistent Schrödinger-Poisson simulations [32] of the device stack, closely matching results of the equivalent capacitor model [see Fig. 1(a)], accurately reproduce this experimental bias dependence of electron density requiring, e.g., at $\mathrm{V}_{\mathrm{BG}}=0.5 \mathrm{~V}$, about $0.136 \mathrm{~V}$ of additional top gate bias per LL versus $0.134 \mathrm{~V}$ per LL measured.

To gain better understanding of the interplay between the edge and bulk conductances in multiport devices of complex geometries, a 2D resistor network was modeled, parametrized by linear edge and bulk resistivities: $\rho_{\text {edge }}(\Omega / \mu \mathrm{m})$ and $\rho_{\text {bulk }}(\Omega / \square)$ (See Supplemental Material [24], Sec. III). Results of this numerical extraction are shown in Fig. 3, both in the absence of the magnetic field and at $B=2 \mathrm{~T}$ (additional extracted maps for the case of $B=1 \mathrm{~T}$ are shown in supporting Fig. S9).

As expected, at $B=0 \mathrm{~T}$, the extraction procedure is meaningful only near the RPs. Further outside of the RPs, the channel resistance, governed by the diminishing bulk resistivity, becomes so small that voltage signals measured at the 3-4 and 5-6 leads are in the noise level, preventing further quantification of $\rho_{\text {bulk }}$ and $\rho_{\text {edge }}$ (beyond knowing that $\rho_{\text {bulk }}$ is smaller than its noise-level bound). In the gate bias domain allowing for quantitative extraction, the obtained $\rho_{\text {bulk }}$ is a sharp function of gate biases spanning more than 3 orders of magnitude, while $\rho_{\text {edge }}$ is relatively flat (all the way up to the domain edges).

Under a magnetic field, the enhanced bulk resistance outside the RPs (also accompanied by introduction of strong additional edge conduction of different nature) made the signal at the 3-4 and 5-6 leads measureable, thus facilitating the reliable numerical extraction over the whole 2D map. The crossover canyon at the RP boundary, mentioned above, is present again in the bulk resistivity map [Fig. 3(c)] along with a large "step" in $\rho_{\text {edge }}\left[\right.$ Fig. 3(d)]. The extracted $\rho_{\text {bulk }}$ and $\rho_{\text {edge }}$ maps both show correlated ripplings due to LL filling, as expected: when Fermi level falls halfway between LLs, bulk turns most resistive while, simultaneously, IQHE edge channels are most protected and, consequently, most conductive. Indeed, the ripplings in $\rho_{\text {bulk }}$ and $\rho_{\text {edge }}$ are almost in antiphase as can be best seen by examining the upper-right corner of two maps in Figs. 3(c) and 3(d).

The same extraction procedure was performed on data collected at different temperatures, revealing the decoupled temperature dependence for bulk and edge resistivities. Similar to the temperature dependent trend for resistances in Fig. S2(a), S2(b), the bulk resistivity [Fig. S2(d)]
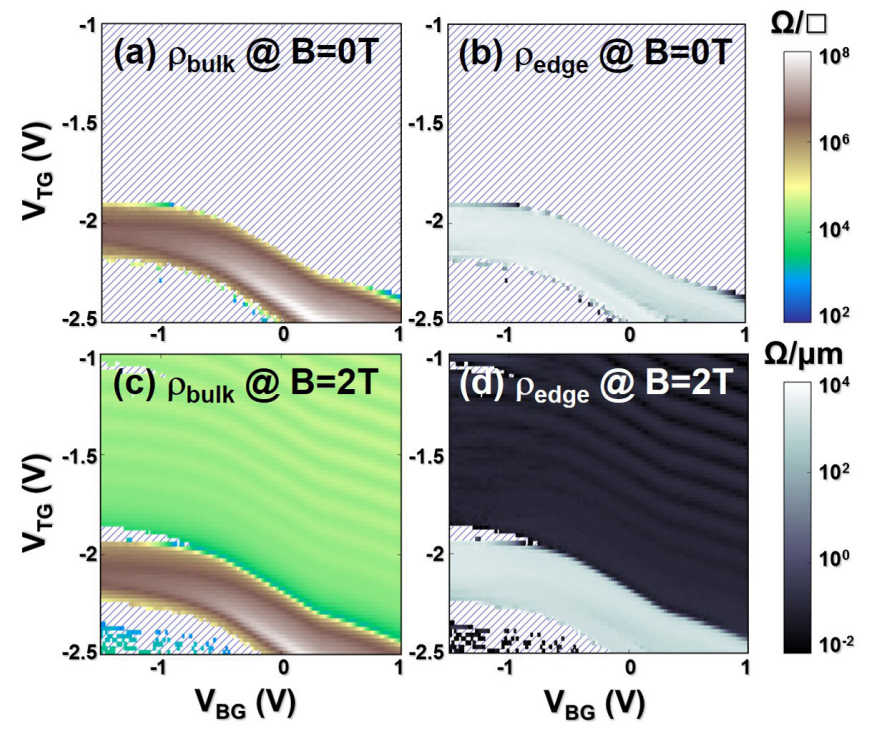

FIG. 3. 2D maps of extracted bulk and edge resistivities at $1.8 \mathrm{~K}$ : (a), (b) at $B=0$ and (c),(d) at $B=2 \mathrm{~T}$.

depends exponentially on temperature, and the energy gap is closing when going from A to E. The edge resistivity [Fig. S2(c)] stays at $\sim 2 \mathrm{k} \Omega / \mu \mathrm{m}$, which is consistent with the values extracted above from edge dominated resistance. This resistivity is insensitive to the measured temperatures between 1.8 and $25 \mathrm{~K}$. It is important to note that at this resistivity level, a mesoscopic Hall bar with lengths of a few micrometers can easily attain a resistance close to the theoretically predicted quantized conductance purely by coincidence. Thus measuring a non-local conductance near the expected quantized value is not necessarily a solid proof of helical edge transport in the inverted regime.

The ability to independently map the bulk and edge resistivities in a $2 \mathrm{D}$ top-back gate bias space allows for a
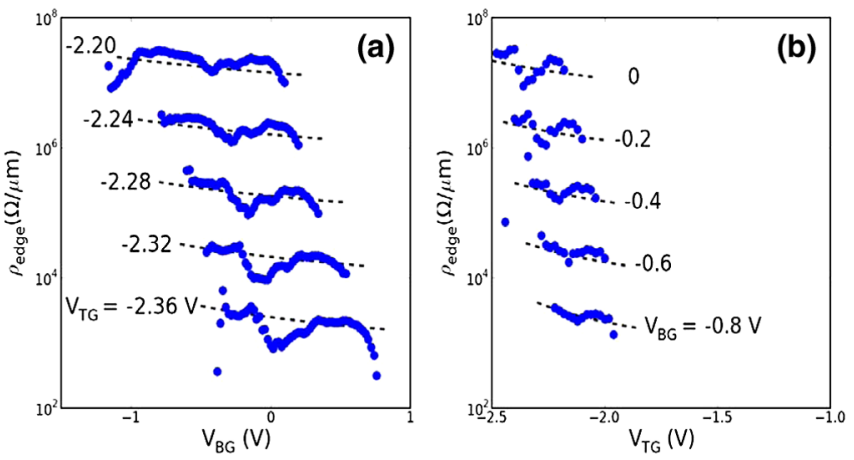

FIG. 4. Dots in (a) and (b) represent 1D line cuts along $V_{\mathrm{BG}}$ and $V_{\mathrm{TG}}$ directions, respectively, through the 2D map of the extracted edge resistivity $[B=0$ case, Fig. 3(b)]. The lowest cut in each subfigure is shown with its true value, while each consecutive cut is offset vertically by a factor of 10 . All dashed lines collectively represent a simple three-parameter fit to the extracted data using an expansion of edge conductivity $1 / \rho_{\text {edge }}$ up to the first order in $\left(V_{\mathrm{BG}}, V_{\mathrm{TG}}\right)$. Using this global fit, modulation of edge conductance across the domain of reliable extraction exceeds $2 \mathrm{x}$. 
further assessment on the nature of the edge channel. Shown in Fig. 4(a) and 4(b) are 1D line cuts of the edge resistivity as a function of back and top gate biases, respectively. In the regime where extraction is reliable (high bulk resistivity), the edge resistivity exhibits a negative slope with respect to both top and back gates. This signifies higher edge conductance with more positive gate bias, which is a behavior of $n$-type transport. The electronlike behavior of the edge conductance can be explained by the well-known Fermi level pinning and electron accumulation at the surface of InAs [27-31]. It is important to note that this trend for edge resistivity is resolved well beyond the numerical uncertainty of the extraction method. Indeed, the same trend can also be observed in the raw measurement resistance data for conductance paths that directly involve edge channels, like the $R_{1-3 ; 1-3}$ of the Corbino ring (See Fig. S4), or longitudinal resistance of a Hall bar (See Fig. S5).

In summary, we have shown evidence that the trivial regime of InAs/GaSb double-quantum-well structures can host an $n$-type edge conductance channel. The Corbino ring geometry provided a simple device prototype that enables analysis of the interplay between the bulk and edge conduction channels, and, together with the resistor network model, offered a template for independent quantification of the bulk and edge conductivities. The ability to modulate the edge conductance with gate biases opens hope to controllably suppress the trivial edge conductance and reveal the helical topological edge states in future investigations.

This work was partially supported by Microsoft Station Q, the Netherlands Foundation for Fundamental Research on Matter (FOM), the Danish National Research Foundation, and the European Commission through the Marie Curie Fellowship Program.

*mbnguyen@hrl.com †Sokolich@hrl.com

[1] Y. Naveh and B. Laikhtman, Appl. Phys. Lett. 66, 1980 (1995).

[2] S. de-Leon, L. D. Shvartsman, and B. Laikhtman, Phys. Rev. B 60, 1861 (1999).

[3] M. J. Yang, C. H. Yang, B. R. Bennett, and B. V. Shanabrook, Phys. Rev. Lett. 78, 4613 (1997).

[4] M. Drndic, M. P. Grimshaw, L. J. Cooper, D. A. Ritchie, and N. K. Patel, Appl. Phys. Lett. 70, 481 (1997).

[5] L. J. Cooper, N. K. Patel, V. Drouot, E. H. Linfield, D. A. Ritchie, and M. Pepper, Phys. Rev. B 57, 11915 (1998).

[6] K. Suzuki, K. Takashina, S. Miyashita, and Y. Hirayama, Phys. Rev. Lett. 93, 016803 (2004).

[7] C. Liu, T. L. Hughes, X.-L. Qi, K. Wang, and S.-C. Zhang, Phys. Rev. Lett. 100, 236601 (2008).

[8] S. Mi, D. I. Pikulin, M. Wimmer, and C. W. J. Beenakker, Phys. Rev. B 87, 241405 (2013).

[9] F. Qu, A. J. A. Beukman, S. Nadj-Perge, M. Wimmer, B.-M. Nguyen, W. Yi, J. Thorp, M. Sokolich, A. A. Kiselev, M. J.
Manfra, C. M. Marcus, and L. P. Kouwenhoven, Phys. Rev. Lett. 115, 036803 (2015).

[10] I. Knez, PhD thesis, Rice University, 2012.

[11] F. Nichele, A. N. Pal, P. Pietsch, T. Ihn, K. Ensslin, C. Charpentier, and W. Wegscheider, Phys. Rev. Lett. 112, 036802 (2014).

[12] B.-M. Nguyen, W. Yi, R. Noah, J. Thorp, and M. Sokolich, Appl. Phys. Lett. 106, 032107 (2015).

[13] I. Knez, R.-R. Du, and G. Sullivan, Phys. Rev. Lett. 107, 136603 (2011).

[14] K. Suzuki, Y. Harada, K. Onomitsu, and K. Muraki, Phys. Rev. B 87, 235311 (2013).

[15] I. Knez, C. T. Rettner, S.-H. Yang, S. S.-P. Parkin, L. Du, R.-R. Du, and G. Sullivan, Phys. Rev. Lett. 112, 026602 (2014).

[16] L. Du, I. Knez, G. Sullivan, and R.-R. Du, Phys. Rev. Lett. 114, 096802 (2015).

[17] S. Mueller, A. N. Pal, M. Karalic, T. Tschirky, C. Charpentier, W. Wegscheider, K. Ensslin, and T. Ihn, Phys. Rev. B 92, 081303 (2015).

[18] K. Suzuki, Y. Harada, K. Onomitsu, and K. Muraki, Phys. Rev. B 91, 245309 (2015).

[19] V. S. Pribiag, A. J. A. Beukman, F. Qu, M. C. Cassidy, C. Charpentier, W. Wegscheider, and L. P. Kouwenhoven, Nat. Nanotechnol. 10, 593 (2015).

[20] E. M. Spanton, K. C. Nowack, L. Du, G. Sullivan, R.-R. Du, and K. A. Moler, Phys. Rev. Lett. 113, 026804 (2014).

[21] D. I. Pikulin and T. Hyart, Phys. Rev. Lett. 112, 176403 (2014).

[22] F. Nichele, H. J. Suominen, M. Kjaergaard, C. M. Marcus, E. Sajadi, J. A. Folk, F. Qu, A. J. A. Beukman, F. K. d. Vries, J. v. Veen, S. Nadj-Perge, L. P. Kouwenhoven, B.-M. Nguyen, A. A. Kiselev, W. Yi, M. Sokolich, M. J. Manfra, E. M. Spanton, and K. A. Moler New J. Phys. 18, 083005 (2016).

[23] C. Charpentier, S. Fält, C. Reichl, F. Nichele, A. Nath Pal, P. Pietsch, T. Ihn, K. Ensslin, and W. Wegscheider, Appl. Phys. Lett. 103, 112102 (2013).

[24] See Supplemental Material at http://link.aps.org/ supplemental/10.1103/PhysRevLett.117.077701 for description of the resistor network model and additional measurement data (temperature dependent, local vs. nonlocal transport), which includes Refs. [9,12,16,25,26].

[25] M. J. Manfra, E. H. Hwang, S. Das Sarma, L. N. Pfeiffer, K. W. West, and A. M. Sergent, Phys. Rev. Lett. 99, 236402 (2007)

[26] G. Chen, A. M. Hoang, S. Bogdanov, A. Haddadi, P. R. Bijjam, B.-M. Nguyen, and M. Razeghi, Appl. Phys. Lett. 103, 033512 (2013).

[27] C. A. Mead and W. G. Spitzer, Phys. Rev. Lett. 10, 471 (1963).

[28] J. M. Woodall, J. L. Freeouf, G. D. Pettit, T. Jackson, and P. Kirchner, J. Vac. Sci. Technol. 19, 626 (1981).

[29] M. Noguchi, K. Hirakawa, and T. Ikoma, Phys. Rev. Lett. 66, 2243 (1991).

[30] D. C. Tsui, Phys. Rev. Lett. 24, 303 (1970).

[31] L. Ö. Olsson, C. B. M. Andersson, M. C. Håkansson, J. Kanski, L. Ilver, and U. O. Karlsson, Phys. Rev. Lett. 76, 3626 (1996).

[32] W. Yi, A. A. Kiselev, J. Thorp, R. Noah, B.-M. Nguyen, S. Bui, R. D. Rajavel, T. Hussain, M. F. Gyure, P. Kratz, Q. Qian, M. J. Manfra, V. S. Pribiag, L. P. Kouwenhoven, C. M. Marcus, and M. Sokolich, Appl. Phys. Lett. 106, 142103 (2015). 